\title{
Adherence to Outpatient Oral Medication Regimens in Adolescent HSCT Recipients
}

\author{
Meghan E. McGrady, Ph.D. ${ }^{1}$, Sarah N. Williams, B.S. ${ }^{1}$, Stella M. Davies, MBBS, Ph.D., \\ MRCP $^{2}$, and Ahna L.H. Pai, Ph.D. ${ }^{1}$ \\ ${ }^{1}$ Center for the Promotion of Treatment Adherence and Self-Management, Division of Behavioral \\ Medicine and Clinical Psychology, Cincinnati Children's Hospital Medical Center, Cincinnati, Ohio \\ 2Division of Bone Marrow Transplantation and Immune Deficiency, Cincinnati Children's Hospital \\ Medical Center, Cincinnati, Ohio
}

\begin{abstract}
Purpose-Hematopoietic stem cell transplantation (HSCT) is an increasingly utilized treatment option for adolescents with many life-threatening diagnoses. Suboptimal adherence may result in compromised treatment effectiveness and increased risk of adverse medical outcomes.

Method-This study examined adherence patterns in six adolescents (ages 12-18) who had undergone HSCT. Demographic and clinical information were obtained from caregivers and via chart review. Electronic pill bottles (Medical Event Monitors, MEMS ${ }^{\mathrm{TM}}$ ) were used to track medication adherence. Daily, weekly, and monthly adherence as well as medication interruptions (periods of $\geq 24$ hours between doses) were calculated.
\end{abstract}

Results-Participants took $73 \%$ of doses $(\mathrm{SD}=13 \%)$ and demonstrated perfect adherence on $56 \%$ of days $(\mathrm{SD}=18 \%$, Range $=34-88 \%)$. Average monthly adherence ranged from $40-91 \%$ and decreased over time. Participants demonstrated at least two $[\mathrm{M}(\mathrm{SD})=4.20(2.28)]$ medication interruptions. Individual adherence patterns included high sustained adherence, variable adherence, and delayed non-adherence.

Conclusions-Overall, participants struggled to adhere to medication schedules, taking less than three-quarters of prescribed doses and demonstrating perfect adherence on fewer than four out of seven days per week. Adherence rates are similar to those observed in other pediatric populations and demonstrate the importance of routinely assessing adherence in adolescents who have undergone HSCT.

\section{Keywords}

Adolescent; Adherence; Hematopoietic stem cell transplantation

\footnotetext{
(C) 2013 Elsevier Ltd. All rights reserved.

Corresponding Author: Meghan E. McGrady, Ph.D. Center for the Promotion of Treatment Adherence and Self-Management, Cincinnati Children's Hospital Medical, Cincinnati, OH 45229-3039, USA. Meghan.McGrady@cchmc.org Phone: (01) 513-803-8044 Fax: (01) 513-803-0415.

Conflict of Interest Statement

None declared

Publisher's Disclaimer: This is a PDF file of an unedited manuscript that has been accepted for publication. As a service to our customers we are providing this early version of the manuscript. The manuscript will undergo copyediting, typesetting, and review of the resulting proof before it is published in its final citable form. Please note that during the production process errorsmaybe discovered which could affect the content, and all legal disclaimers that apply to the journal pertain.
} 
Driven by advances in research and clinical trials, hematopoietic stem cell transplantation (HSCT), a treatment once considered more beneficial for younger children, is now considered an effective treatment option for adolescents with oncological, hematological, and immunological diseases (Dini et al., 2011, Savaşan and Abella, 2005). Between 2008 and 2010, approximately a third of pediatric HSCT recipients were between 10 and 19 years of age (National Marrow Donor Program, 2013). While survival rates for adolescents who have undergone HSCT have significantly improved, three year survival rates demonstrate wide variability and range from approximately $35-93 \%$ depending on the diagnosis (National Marrow Donor Program, 2012). In addition to numerous medical factors, adherence, or the "extent to which a person's behavior (i.e., taking medications) coincides with medical or health advice", is a modifiable factor that likely accounts for variation in these outcomes (Haynes, Taylor, \& Sackett, 1979).

Following HSCT, adolescents and their caregivers must manage a complex treatment regimen. This regimen often includes the administration of numerous intravenous and/or oral medications with different dosing schedules and frequent dosing changes. In addition, adolescents and their caregivers are often asked to attend three to four clinic appointments each week and abide by activity restrictions (i.e., avoiding crowded places). As more complex medical regimens are associated with poorer adherence, individuals who must adhere to the post-HSCT regimen are at risk for non-adherence (Coleman et al., 2012).

Within this at-risk population, non-adherence may be particularly problematic for adolescents. Across numerous medical conditions, including many for which HSCT is the treatment of choice, adolescents demonstrate the highest rates of non-adherence (Butow et al., 2010, Pritchard et al., 2006). Rates of non-adherence among adolescents with cancer, for example, range from 27-63\% (Butow et al., 2010). If rates of non adherence in adolescents who have undergone HSCT are similar to those demonstrated by other populations, a significant proportion of adolescents may be receiving sub-therapeutic doses of medication, compromising treatment effectiveness and placing them at increased risk for treatment failure and premature death (Pai and Drotar, 2010). Given these significant health implications and the modifiable nature of non-adherence, it is important to understand the prevalence of non-adherence in adolescent HSCT recipients.

The only investigation of adherence in HSCT recipients to date indicated that $40 \%$ of adolescents evidenced significant difficulty adhering to their oral antibiotic regimen during hospitalization (Phipps and DeCuir-Whalley, 1990). While qualitative findings suggest similar difficulties are present following discharge (Cooke et al., 2011), studies have not yet examined adherence during this difficult transition. Conceptualization of the post- HSCT regimen within the developmental framework of adolescence, however, suggests that adherence following discharge may be particularly problematic.

Following discharge, normative developmental goals of adolescence collide with the demands of the post-HSCT treatment regimen. Adolescents negotiate increasing independence and new social roles, resulting in unpredictable schedules, decreased parental supervision, increased parent-child conflict, and increased time away from home. In other adolescent chronic illness populations, these normative transitions increase the likelihood that adolescents will forget or choose to forgo treatment tasks (Butow et al., 2010, Pritchard et al., 2006). For example, it is normative for adolescents to place increased importance on peer relationships, making the social acceptance and enjoyment of engaging in social activities far more reinforcing than following a restrictive medical regimen.

Key barriers to adherence (i.e., conflict with caregivers, psychological disorders) also increase during adolescence (Kyngäs et al., 2000). The more barriers faced by adolescents 
with a chronic condition, the higher the rates of non-adherence (Bregnballe et al., 2011, Gray et al., 2012). As a result of these risk factors, it is likely that rates of non-adherence in adolescent HSCT recipients following discharge may be even higher than the rate of $40 \%$ demonstrated during hospitalization (Phipps and DeCuir-Whalley, 1990).

Understanding medication non-adherence in this at-risk population is essential for determining methods to promote adherence to a daunting treatment regimen. The aims of this study were to be the first to describe individual and cohort-level patterns of oral medication adherence over a six- to nine-month period. We hypothesized that adherence would be similar to previously published oral medication adherence rates for adolescents with solid organ transplants or cancer (Butow et al., 2010, Dobbels et al., 2010).

\section{Materials and Methods \\ Participants and Procedures}

Data for this study are from a larger project examining adherence in pediatric HSCT recipients (Pai et al., 2011). Ninety-one of the 119 eligible participants agreed to participate (76\% recruitment rate). The retention rate for the larger study was $99 \%$.

The eight participants (ages 12-18 years) in the current study were recipients of a HSCT, prescribed oral medication, living with a caregiver, and fluent in English. Exclusion criteria included significant cognitive deficits (in the patient or both caregivers) and a medical status that precluded questionnaire completion.

Caregivers completed a questionnaire and were provided with an electronic pill bottle for use following discharge. Data were downloaded from pill bottles at four time points (1-, 3-, 6-, and 9-months). All procedures were approved by the Institutional Review Board and appropriate consent and assent were obtained.

\section{Measures}

Caregivers provided demographic information. Date of transplant, hospitalizations, and changes in medications were obtained via chart review. Immunosuppressant medication adherence was assessed using Medication Event Monitors (MEMS ${ }^{\mathrm{TM}}$ ), an electronic pill bottle that time-stamps each bottle opening. Participants were asked to use MEMS ${ }^{\text {TM }}$ devices to store and administer their immunosuppressant medication for the nine-month study period. Medications stored in the MEMS bottles at baseline included: Cyclosporine ( $\mathrm{n}$ $=3)$, Fluconasol $(\mathrm{n}=1)$, Acyclovir $(\mathrm{n}=1)$, and Voriconazole $(\mathrm{n}=1)$. When immunosuppressant prescriptions were changed, participants were instructed to change the medications stored in the MEMS ${ }^{\mathrm{TM}}$ bottle. All participants evidenced at least one change in monitored immunosuppressant medication during the course of the study, and at study completion, medications in the MEMS ${ }^{\mathrm{TM}}$ bottles included: Acyclovir $(\mathrm{n}=3)$, Prograf $(\mathrm{n}=$ $2)$, and Bactrim $(\mathrm{n}=1)$.

\section{Analyses}

Two participants were excluded from analyses (loss of device, $\mathrm{n}=1$; discontinued use of device within 40 days, $\mathrm{n}=1$ ), resulting in a final sample of six participants. A-priori decision rules were applied to account for times when medications were not being dispensed from the bottle. Periods during which a patient was hospitalized, in the emergency room, or having a medication "held" by the medical team were coded as "non-monitored." Data were also coded as "non-monitored" if families reported they were no longer using the MEMS ${ }^{\mathrm{TM}}$ bottle. 
Daily adherence was calculated by dividing the number of pill bottle openings per day by the number of prescribed doses for that day. Weekly and monthly adherence values were calculated by computing the mean of daily adherence values across the specified time frame. The frequency and average length of medication interruptions ( $\geq 24$ hours between doses) were calculated for all participants prescribed daily medication $(n=5)$.

\section{Results}

Participants were primarily male (67\%), Caucasian (83\%), and non-Hispanic (83\%). On average, participants were $14.09(\mathrm{SD}=1.67)$ years of age and $26.83(\mathrm{SD}=10.93)$ days posttransplant at baseline. Medical diagnoses included: acute lymphoblastic leukemia (ALL, $\mathrm{n}=$ 2, 33\%), Fanconi Anemia ( $\mathrm{n}=1,17 \%)$, Medulloblastoma $(\mathrm{n}=1,17 \%)$, Hodgkin's Lymphoma $(\mathrm{n}=1,17 \%)$, and acute myeloid leukemia (AML, $\mathrm{n}=1,17 \%)$.

\section{Adherence}

Five participants (83\%) reliably utilized MEMS ${ }^{\mathrm{TM}}$ for the duration of their immunosuppressant medication prescription ( 3 for 9 months, 1 for 4 months, and 1 for 7 months). Six months of data are also included for one patient who did not provide their MEMS bottle after the 6-month visit. Electronic monitor data were available for an average of 174.50 days per participant $(\mathrm{SD}=67.19$ days, Range $=110-268$ days).

On average, participants took $73 \%$ of prescribed doses $(\mathrm{SD}=13 \%$, Range $=55-92 \%)$. Average number of doses taken per month ranged from $40 \%(\mathrm{SD}=26 \%)$ to $91 \%(\mathrm{SD}=$ $13 \%)$ and decreased over time. Standard deviations (Range $=13 \%-33 \%)$ indicated that adherence varied widely across participants.

Mean percentage of days with perfect adherence (correct number of doses taken) was $56 \%$ ( $\mathrm{SD}=18 \%$, Range $=34-88 \%)$. On average, participants failed to take all prescribed doses on $13 \%$ of days $(\mathrm{SD}=14 \%$, Range $=1-37 \%$ ). All participants taking daily medication demonstrated at least 2 medication interruptions (periods of $\geq 24$ hours between doses). On average, participants demonstrated 4.20 interruptions $(\mathrm{SD}=2.28$, Range $=2-7)$ lasting an average of 4.04 days each $(\mathrm{SD}=4.60$, Range $=1.5-12$ days $)$. Weekly adherence data for each participant are presented in Figure 1.

\section{Individual Adherence Patterns}

Participant 1 (P1) was a 12 year-old boy prescribed once-a-day Fluconasol at discharge. For the first 8 months of the study, P1 demonstrated variable weekly adherence ranging from 50-100\%. After 31 weeks, P1 demonstrated a significant decline in adherence, taking no doses for several weeks. Examination of the electronic medical record did not identify any potential contributing factors (i.e., hospitalizations, changes in medications).

Participant 2 (P2) was a 13 year-old female prescribed three-times-daily Acyclovir at discharge. Similar to P1, P2 demonstrated variable adherence during the first few months of the study, with weekly adherence percentages ranging from $25-95 \%$. Following a hospitalization three months after study entry, P2 demonstrated poor weekly adherence (0$7 \%$ ) for several weeks. After her medication was changed from a twice-daily dosing schedule to once-a-day, $\mathrm{P} 2$ demonstrated significant improvement in her immunosuppressant adherence, averaging $100 \%$ weekly adherence for the remainder of the study.

Participant 3 (P3) was a 13 year-old female originally prescribed twice-a-day Cyclosporine. For the first five months of the study, P3 demonstrated consistently high weekly adherence (75-100\%). Directly following a change to 3 times daily Acyclovir at 21 weeks, P3 began to 
demonstrate lower levels of adherence (38-76\%) that persisted for the remainder of the study.

Participant 4 (P4) was a 16 year-old male originally prescribed twice-daily Cyclosporine. While he evidenced good adherence initially (weekly average $>80 \%$ for 8 weeks), over time, $\mathrm{P} 4$ demonstrated a steadily decreasing pattern of adherence. Of note, the decreases in $\mathrm{P} 4$ 's adherence did not appear to correspond to changes in his medication regimen or hospitalizations.

Participant 5 (P5) was a 16 year-old male prescribed twice-daily Cyclosporine. P5 demonstrated consistently high weekly adherence (85-100\%) for the first 23 weeks of the study. After discontinuing Cyclosporine and beginning Acyclovir three times daily at week 24 , P5 began to evidence declines in adherence (42-100\%). Of note, P5 did not return to the higher levels of adherence he evidenced at the beginning of the study.

Participant 6 (P6) was a 13 year-old male prescribed twice-daily Voriconazole. P6 demonstrated fairly consistent patterns of weekly adherence (57-100\%) throughout the duration of the study.

\section{Discussion}

Adolescent HSCT recipients in this study demonstrated considerable non-adherence, taking an average of $73 \%$ of prescribed doses. Substantial variability in adherence was present within and across adolescents. Patterns of adherence included high sustained adherence, variable adherence, and delayed non-adherence. Results of individual-level data suggest that changes in medication regimens may partially contribute to changes in adherence over time.

Despite the critical health status of these adolescents and the significant implications of nonadherence, adherence patterns were similar to those observed in other pediatric populations (Butow et al., 2010, Dobbels et al., 2010, Pritchard et al., 2006). This is contrary to the often-held notion that their significant medical history will motivate adolescents who receive HSCTs to be adherent.

Adolescents missed at least one dose of medication about three days a week. This high number of missed doses inevitably impacts medication exposure, compromises treatment effectiveness, and could potentially increase the risk of adverse medical outcomes. These findings are troubling since problematic adherence is associated with an increased likelihood of HSCT-related mortality among adults (Pereira et al., 2010). Adherence difficulties became even more pronounced over time, decreasing from $91 \%$ during the first month following discharge to less than $80 \%$ after 3 months and less than $60 \%$ after 6 months. Consistent with previous research (Modi et al., 2011), these declines highlight the difficulty of maintaining a complex medical regimen.

The high prevalence of non-adherence in this study supports detailed and objective assessment of medication-taking patterns. Clinicians may wish to consider electronic monitoring devices similar to those used in this study (Ingerski et al., 2011). In addition, the variability in adherence across participants suggests that patient-centered, individualized adherence-promotion efforts may be most effective. Some patients may require intensive intervention efforts prior to and directly following discharge, while positive reinforcement and screening may be more appropriate for others.

As with most nascent areas of research, the generalizability of this study is limited by its small sample size. However, as the larger study had a recruitment rate of $83 \%$, the participants in this study represent a large majority of adolescent HSCT recipients at one 
pediatric hospital. In addition, electronic monitors represent a proxy for medication taking and the actual ingestion of the medication was not confirmed. Third, patients were not prescribed identical immunosuppressant regimens, and these differences may have impacted adherence patterns. Finally, the correlational nature of the data limits our ability to draw causal inferences. Future studies should include larger samples, include additional measures of adherence and examine potential predictors of adherence using qualitative and quantitative methods. Ultimately, randomized controlled trials are needed to determine variables that lead to changes in adherence and health outcomes among adolescent HSCT recipients.

This study is the first to examine adherence in adolescent HSCT recipients. Adolescents in this sample demonstrated substantial non-adherence consistent with rates of other pediatric populations. Additional research assessing the generalizability of these findings and the predictors and outcomes of non-adherence is needed. If replicated, these findings support the development of methods to assess and promote adherence and, ultimately, health outcomes of these courageous patients.

\section{Acknowledgments}

\section{Funding Sources}

This research is supported in part by grant T32HD068223 for M.M.

\section{References}

Bregnballe V, Schiotz PO, Boisen KA, Pressler T, Thastum M. Barriers to adherence in adolescents and young adults with cystic fibrosis: a questionnaire study in young patients and their parents. Patient Preferences and Adherence. 2011; 5:507-515.

Butow P, Palmer S, Pai A, Goodenough B, Luckett T, King M. Review of adherence-related issues in adolescents and young adults with cancer. Journal of Clinical Oncology. 2010; 28 (32):4800-4809. [PubMed: 20212260]

Coleman CI, Limone B, Sobieraj DM, Lee S, Roberts MS, Kaur R, Alam T. Dosing frequency and medication adherence in chronic disease. Journal of Managed Care Pharmacy. 2012; 18(7):527_ 539. [PubMed: 22971206]

Cooke L, Chung C, Grant M. Psychosocial care for adolescent and young adult hematopoietic cell transplant patients. Journal of Psychosocial Oncology. 2011; 29 (4):394-414. [PubMed: 21966725]

Dini G, Zecca M, Balduzzi A, Messina C, Masetti R, Fagioli F, Favre C, Rabusin M, Porta F, Biral E, Ripaldi M, Iori AP, Rognoni C, Prete A, Locatelli F. No difference in outcome between children and adolescents transplanted for acute lymphoblastic leukemia in second remission. Blood. 2011; 118 (25):6683-6690. [PubMed: 22010101]

Dobbels F, Ruppar T, De Geest S, Decorte A, Van Damme-Lombaerts R, Fine RN. Adherence to the immunosuppressive regimen in pediatric kidney transplant recipients: A systematic review. Pediatric Transplantation. 2010; 14 (5):603-613. [PubMed: 20214741]

Gray WN, Denson LA, Baldassano RN, Hommel KA. Treatment adherence in adolescents with inflammatory bowel disease: the collective impact of barriers to adherence and anxiety/depressive symptoms. Journal of Pediatric Psychology. 2012; 37 (3):282-291. [PubMed: 22080456]

Haynes, RB.; Taylor, DW.; Sackett, DL. Compliance in Health Care. Baltimore: Johns Hopkins University Press; 1979. Determinants of compliance: The disease and mechanics of treatment.

Ingerski LM, Hente EA, Modi AC, Hommel KA. Electronic measurement of medication adherence in pediatric chronic illness: A review of measures. Journal of Pediatrics. 2011; 159 (4):528-534. [PubMed: 21722917]

Kyngäs HA, Kroll T, Duffy ME. Compliance in adolescents with chronic diseases: A review. Journal of Adolescent Health. 2000; 26 (6):379-388. [PubMed: 10822178] 
Modi AC, Rausch JR, Glauser TA. Patterns of nonadherence to antiepileptic drug therapy in children with newly diagnosed epilepsy. JAMA. 2011; 305 (16):1669-1676. [PubMed: 21521848]

National Marrow Donor Program. [Accessed July 7, 2013] US transplant centers. 2013. Available at http://bethematch.org/Patient/Transplant_Planning/Choosing_a_Transplant_Center/ Advanced_Search.aspx

National Marrow Donor Program. [Accessed September 26, 2013] A contractor for the C.W. Bill Young Cell Transplantation Program operated through the U. S. Department of Health and Human Services, Health Resources and Services Administration, Healthcare Systems Bureau. Donor Registry Transplant Data. 2012. Available at http://bloodcell.transplant.hrsa.gov/research/ transplant_data/us_tx_data/survival_data/survival.aspx

Pai ALH, Drotar D. Treatment adherence impact: The systematic assessment and quantification of the impact of treatment adherence on pediatric medical and psychological outcomes. Journal of Pediatric Psychology. 2010; 35 (4):383-393. [PubMed: 19710252]

Pai, ALH.; Tackett, A.; Davies, SM. Adherence in hematopoietic stem cell transplant. Presented at the National Conference in Pediatric Psychology; 2011.

Pereira DB, Christian LM, Patidar S, Bishop MM, Dodd SM, Athanason R, Wingard JR, Reddy VS. Spiritual absence and 1-year mortality after hematopoietic stem cell transplant. Biology of Blood Marrow Transplantation. 2010; 16 (8):1171-1179. [PubMed: 20227510]

Phipps S, DeCuir-Whalley S. Adherence issues in pediatric bone marrow transplantation. Journal of Pediatric Psychology. 1990; 15 (4):459-475. [PubMed: 2258795]

Pritchard MT, Butow PN, Stevens MM, Duley JA. Understanding medication adherence in pediatric Acute Lymphoblastic Leukemia: A review. Journal of Pediatric Hematology Oncology. 2006; 28 (12):816-823.

Savaşan S, Abella EM. Current issues in pediatric stem cell transplantation. Clinics in Laboratory Medicine. 2005; 25 (3):519-540. [PubMed: 16129191] 

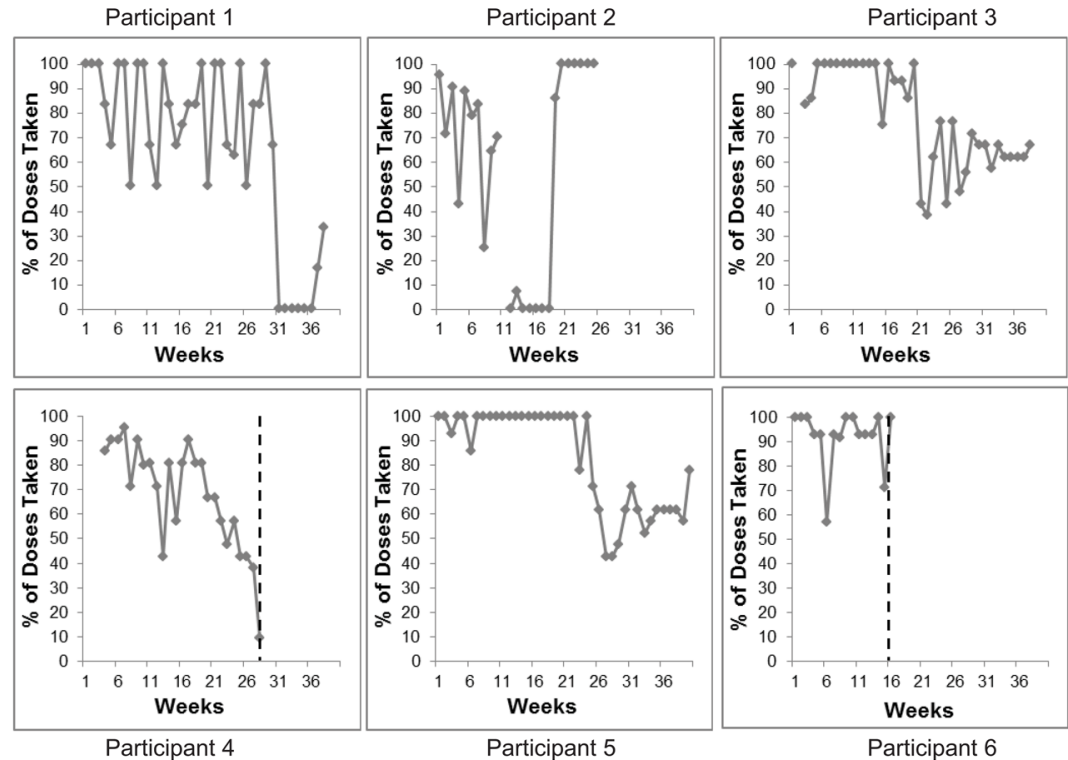

Figure 1.

Weekly Percentage of Doses Taken for Each Participant

Note. Dashed line represents discontinuation of all oral medications. 


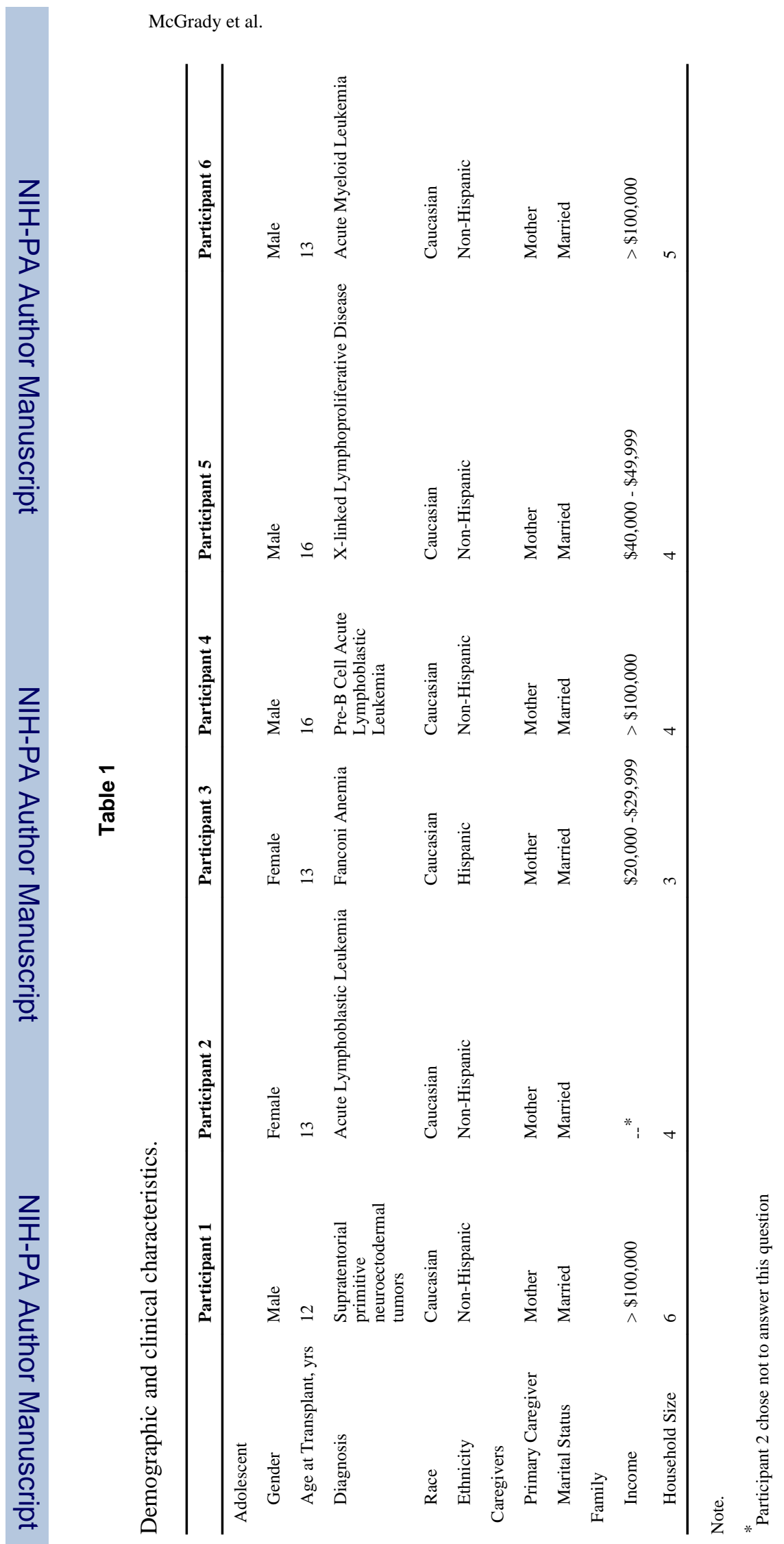

Eur J Oncol Nurs. Author manuscript; available in PMC 2015 April 01. 
